\title{
Isotope ratios determination of uranium in soil samples affected due to Chernobyl accident using thermal ionisation mass spectrometer
}

\author{
S.K. Sahoo, K. Shiraishi, S. Kimura, A. Masuda ${ }^{1}$, I.P. Los ${ }^{2}$ and P.V. Zamostyan ${ }^{2}$ \\ National Institute of Radiological Sciences, Environmental Radiation Protection \\ Research Group, 4-9-1 Anagawa, Inage-ku, Chiba 263-8555, Japan \\ ${ }^{1}$ Department of Chemistry, University of Tokyo, Bunkyo-ku, Tokyo 113-0033, Japan \\ ${ }^{2}$ Research Centre for Radiation Medicine, 53 Melnikova, 254050 Kiev, Ukraine
}

\begin{abstract}
A rapid and highly precise method was developed for the separation of uranium from soil samples for thermal ionisation mass spectrometry. The chemical separation procedure is based on the use of anion exchange resin and UTEVA extraction chromatography column. The isotope ratios of uranium, ${ }^{234} \mathrm{U} /{ }^{238} \mathrm{U},{ }^{235} \mathrm{U} /{ }^{238} \mathrm{U}$ and ${ }^{236} \mathrm{U}^{238} \mathrm{U}$ were measured by using a VG Sector 54 TIMS equipped with a Wide Aperture Retardation Potential (WARP) energy filter with a precision of better than $0.2 \%$ at two standard deviations (sd). The use of a WARP energy filter improves abundance sensitivity by an order of magnitude over a conventional VG Sector TIMS. Replicates of standard reference materials showed excellent analytical agreement with established values, supporting the reliability and accuracy of the method. Uranium isotopic compositions have been determined in soil samples in the exclusion zone of Chemobyl nuclear power plant. The isotopic composition of Chernobyl soil samples showed significant deviation from the natural uranium and the ${ }^{236} \mathrm{U}^{238} \mathrm{U}$ ratio varied from $10^{-5}$ to $10^{-4}$.
\end{abstract}

\section{INTRODUCTION}

Uranium is one of the heaviest naturally occurring element on earth and plays an important role in daily life because of its use in nuclear power plants. Environmental contamination with alpha-emitting nuclides of uranium and transuranium elements is possible during accidents involving nuclear devices or nuclear power plants. As a result of Chernobyl nuclear power plant (CNNP) accident, most of the radioactivity fallout descended in the vicinity of CNPP and adjoining areas of Ukraine and Belarus [1] Thus their characterisation and determination is required for environmental monitoring of large contaminated regions. Due to difference in chemical characteristic of actinides, the migration of uranium differs from the migration of actinides e.g. plutonium. Hence a systematic study of the radionuclides leaching from the fuel particles in soil, which require ultra sensitive isotope analytical methods for direct determination of spent reactor uranium, is important for the control and forecast of the radio-ecological situation in the contaminated areas.

Uranium comprises three natural isotopes with long half-lifes, ${ }^{234} \mathrm{U},{ }^{235} \mathrm{U}$, and ${ }^{238} \mathrm{U}$, the isotopic abundances being approximately of $0.0054 \%, 0.720 \%$ and $99.275 \%$, respectively [2]. These ratios may have small variations depending on the geographic origin of the sample, due to natural isotopic fractionation, nuclear reactions or anthropogenic contamination. The principal isotopes of primordial origin are ${ }^{238} \mathrm{U}$ and ${ }^{235} \mathrm{U}$, with ${ }^{234} \mathrm{U}$ present approximately in radioactive equilibrium with ${ }^{238} \mathrm{U}[3],{ }^{236} \mathrm{U}$ does occur in nature but only at ultra trace concentrations. However, enhanced levels of ${ }^{236} \mathrm{U}$ are produced in nuclear reactors by the neutron irradiation of ${ }^{235} U:{ }^{235} U+{ }^{1} n \rightarrow{ }^{236} U\left(\sigma=98.4\right.$ b) ${ }^{236} U$ is therefore potentially useful as a "fingerprint" for the presence of uranium in the environment which originate from a nuclear reactor. In this regard, it has substantial advantages relative to anthropogenic ${ }^{238} \mathrm{U}$ and ${ }^{235} \mathrm{U}$ since both are quickly diluted by natural uranium background in soils and sediments, making them unsuitable markers for spent uranium released into the environment. In case of environmental sciences, soil and sediments are the most suitable materials that would be likely to preserve any remains of anthropogenically altered uranium. Since soil samples contain high concentration of inherent natural uranium, it is more difficult to detect stnall quantities of $U$ from man made nuclear sources.

In recent days, high precision uranium isotopic measurements are in rapid process using multicollector inductively coupled plasma mass spectrometry (MC-ICP-MS) [4] and thermal ionisation mass spectrometry (TIMS) equipped with energy filters [5]. Since the natural ${ }^{236} \mathrm{U} /{ }^{238} \mathrm{U}$ abundance ratio is 
extremely low, arising from activation processes as cosmic ray activation, mass spectrometri instruments capable to measure isotope abundance ratios within a dynamic range of $8-10$ orders of magnitude is required. Recently, extraction chromatographic separation method has been found more suitable over anion exchange chromatography or liquid-liquid extraction method for the separation of uranium from the sample to reduce a large volume of waste.

This paper reports the result of measurement of ${ }^{234} \mathrm{U} /{ }^{238} \mathrm{U},{ }^{235} \mathrm{U} /{ }^{238} \mathrm{U}$ and ${ }^{236} \mathrm{U} /{ }^{238} \mathrm{U}$ ratios from soil samples in Chernobyl exclusion zones using a thermal ionization mass spectrometry equipped with a WARP energy filter to detect minute levels of enriched or depleted uranium.

\subsection{EXPERIMENTAL}

\subsubsection{Instrument}

The isotopic analyses of uranium were measured on a single focussing VG (Micromass) Sector $\$ 4$ thermal ionization mass spectrometer (TIMS) at National Institute of Radiological Sciences. The instrument is equipped with nine Faraday cup collectors and a Daly ion-counting system detector positioned behind axial Faraday and wide aperture retardation potential (WARP) energy filter. Signals from the Daly detector are received by an EG \& G Ortec 9302 amplifier / discriminator and EG \& $G$ Ortec 996 ion counter/timer.

\subsubsection{Reagents and Standards}

High purity water was obtained with a Millipore Milli-Q water purification system. High purity HCl, $\mathrm{HNO}_{3}, \mathrm{HF}$ and $\mathrm{HClO}_{4}$ were purchased from Tamapure chemical industriers (Kawasaki, Japan). The strong anion exchange resin Dowex 1X-8 (200-400 mesh, $\mathrm{Cl}^{-}$form) was purchased from Bio-Rad Lats (Richmond, CA). It was soaked in $6 \mathrm{M} \mathrm{HNO}_{3}$ and subsequently washed with distilled water. Commercial extraction chromatographic resin, UTEVA was purchased from Eichrom Industries (Darien, IL) and consists of a neutral organophosphorus diamyl amyl phosphonate extractant adsorbed onto an inet polyacrylamide support. It was conditioned with $7 \mathrm{M} \mathrm{HNO}_{3}$.

\subsubsection{Sample Description}

To check the accuracy of the calibration, NBS standards, NBS U010 was used. Isotopic composition of uranium from two uranium ore was analysed for comparison and to detect the presence of ${ }^{2 ?} \mathrm{U}$. Three soil samples were chosen for this study: sediment reference material supplied by Geological Survey of Japan and two surface soil samples from Chernobyl exclusion zone. Sediment sample, JLK-1 and sol samples were digested with $\mathrm{HNO}_{3}, \mathrm{HF}$ and $\mathrm{HClO}_{4}$ in PTFE pressure decomposition vessels A microwave unit (MLS 1200mega, Italy) was used for heating the sample. The samples were evaporated to dryness on a hot plate after digestion. Finally the residues were dissolved in $7 \mathrm{M} \mathrm{HNO}_{3}$ to yield the sample solution for chemical separation.

\subsubsection{Chemical separation.}

We have used a combination of anion exchange and extraction chromatography with a little modification of the earlier methods [6]. First column was prepared by using precleaned anion exchange resins (Dower $1 \mathrm{X}-8,200-400 \mathrm{mesh}, \mathrm{Cl}^{-}$form) and packed into $2 \mathrm{~cm}$ i.d. Pyrex columns up to a height of $6 \mathrm{~cm}$ and the second $1.0 \times 0.5 \mathrm{~cm}$ internal diameter column containing Eichrom UTEVA resin. Both the columnswe conditioned by passing $15 \mathrm{ml}$ of $7 \mathrm{M} \mathrm{HNO}_{3}$. The sample. was transferred to anion exchange column and the eluent was subsequently passed onto the UTEVA column. Both the columns were washed with $10 \mathrm{nil}$ of $7 \mathrm{M} \mathrm{HNO}_{3}$ followed by $20 \mathrm{ml}$ of $3 \mathrm{M} \mathrm{HNO}_{3}$.

The U was eluted from UTEVA column using $5 \mathrm{ml}$ of $0.02 \mathrm{M} \mathrm{HCl}$. Concentration of eluent was 
adjusted to $9 \mathrm{M} \mathrm{HCl}$. The resulting solution was loaded onto an anion exchange column preconditioned with $9 \mathrm{M} \mathrm{HCl}$ and washed with $10 \mathrm{ml}$ of $9 \mathrm{M} \mathrm{HCl}$. Finally, $\mathrm{U}$ was eluted with $10 \mathrm{ml}$ of $0.02 \mathrm{M} \mathrm{HCl}$ and the eluent was evaporated to dryness in a Teflon beaker. Recovery of $U$ from the samples is about $90 \sim 95 \%$.

\subsection{MASS SPECTROMETRY}

A triple filament assembly was used for the thermal ionization of uranium isotopes. The filament material was 5 pass zone refined thenium ribbon ( $\mathrm{H}$. Cross, $99.999 \%$ ) with $0.003 \mathrm{~cm}$ thickness and 0.07 $\mathrm{cm}$ width. Triple Re filaments were prepared by degassing for $1 \mathrm{~h}$ with a $4 \mathrm{~A}$ current under a vacuum better than $5 \times 10^{-6}$ Torr. The isolated $U$ fraction was loaded onto a separable outer filament and heated with a 0.9 A current to leave a dry residue.

The centre filament was heated to produce a ${ }^{187} \mathrm{Re}$ ion current of $0.2 \mathrm{~V}$ and then the side filaments were heated to produce ion current of $2 \times 10^{-11} \mathrm{~A}$ for ${ }^{238} \mathrm{U}$. Uranium masses $234,235,236$ and 238 were measured dynamically using Daly-ion counting and Faraday cup collectors with mass jumps. ${ }^{234} \mathrm{U} /{ }^{238} \mathrm{U}$, ${ }^{235} \mathrm{U}{ }^{238} \mathrm{U}$ and ${ }^{236} \mathrm{U} /{ }^{238} \mathrm{U}$ ratios were determined by static data collection on the Daly-ion counting and Faraday cup collectors. All ratios were taken as the grand mean of 7 blocks of 10 measurements over a period of 80 minutes. The vacuum during data acquisition was better than $1 \times 10^{-8}$ Torr in the flight tube as well as ion source. Accuracy and precision of uranium isotope ratios measurement depends on the linearity of the detection system and mass fractionation of the isotopes during the run.

\subsection{RESULTS \& DISCUSSION}

The reproducibility and accuracy of the method were checked against the analyses of one certified standard reference materials obtained from NBS as NBSU010. The results on our measurement on the uranium reference materials are given in Table 1 . Analytical uncertainties are represented by $\%$ standard errors. The results are in good analytical agreement. Thus, the measurements of the isotopic ratio in the standards demonstrate that isotopic fractionation, if occurs in the system, is less than the uncertainty in the measurement. For the measurement of 20 blocks, each of which is a mean of ten individual isotope ratios, took 151 minutes to complete 20 blocks by using dynamic method. There is not a significant variation of ${ }^{235} \mathrm{U} /{ }^{238} \mathrm{U}$ ratio with time. In case of triple filament assembly, centre filament is very hot which ionizes and the side filaments to evaporate. Since sample is not directly ionized it has been noticed that mass fractionation is slower than that for a single filament [7].

Table 1 Isotopic composition of uranium NIST U010 standard measured by NTRS TIMS

\begin{tabular}{|c|c|c|c|c|c|c|}
\hline \multirow[t]{2}{*}{$\mathrm{N}_{0}$} & \multicolumn{2}{|c|}{${ }^{234} \mathrm{U} /{ }^{238}$} & \multicolumn{2}{|c|}{${ }^{235} \mathrm{U} /{ }^{238}$} & \multicolumn{2}{|c|}{${ }^{236} \mathrm{U} /{ }^{238} \mathrm{U}$} \\
\hline & observed & certified & observed & certified & observed & certfied \\
\hline \multirow[t]{4}{*}{1} & 0.0000545 & \multirow[t]{10}{*}{0.0000546} & 0.0101408 & \multirow[t]{10}{*}{0.010140} & 0.0000694 & \multirow[t]{10}{*}{0.0000687} \\
\hline & \pm 0.042 & & \pm 0.0079 & & \pm 0.051 & \\
\hline & 0.0000547 & & 0.0101428 & & 0.0000695 & \\
\hline & \pm 0.063 & & \pm 0.008 & & \pm 0.057 & \\
\hline \multirow[t]{2}{*}{3} & 0.0000547 & & 0.0101467 & & 0.0000695 & \\
\hline & \pm 0.062 & & \pm 0.0048 & & \pm 0.052 & \\
\hline \multirow{4}{*}{5} & 0.0000546 & & 0.0101407 & & 0.0000691 & \\
\hline & \pm 0.058 & & \pm 0.005 & & \pm 0.056 & \\
\hline & 0.0000545 & & 0.0101414 & & 0.0000689 & \\
\hline & \pm 0.068 & & \pm 0.0062 & & \pm 0.068 & \\
\hline \multirow{2}{*}{$\begin{array}{l}\text { Mear } \\
S D(2\end{array}$} & 0.0000546 & 0.0000546 & 0.0101424 & 0.010140 & 0.0000692 & 0.0000687 \\
\hline & 5) 0.0000002 & & 0.000005 & & 0.0000005 & \\
\hline
\end{tabular}


The measured isotope ratios, ${ }^{234} \mathrm{U} /{ }^{238} \mathrm{U},{ }^{235} \mathrm{U} /{ }^{238} \mathrm{U}$ and ${ }^{236} \mathrm{U} /{ }^{238} \mathrm{U}$, of two uranium ore samples are given in Table 2. The ${ }^{235} \mathrm{U} /{ }^{238} \mathrm{U}$ is is comparable to well-known "normal terrestrial" ratio of about 0.00725 . The original ratio was established in the r-process of nucleosynthesis in a stellar, presolar environment and has then decreased due to $\alpha$-decay since that time, leading to the present terrestinal

Table 2. Isotope ratios of uranium measured from two uranium ores

\begin{tabular}{|c|c|c|c|}
\hline $\begin{array}{l}\text { Sample } \\
\text { Name } \\
\end{array}$ & ${ }^{234} \mathrm{U} /{ }^{238} \mathrm{U}\left( \pm 2 \sigma_{\mathrm{m}}\right)$ & ${ }^{235} \mathrm{U} /{ }^{238} \mathrm{U}\left( \pm 2 \sigma_{\mathrm{m}}\right)$ & ${ }^{236} U / /^{238} U\left( \pm 2 \sigma_{m}\right)$ \\
\hline $\begin{array}{l}\text { Faraday Mine } \\
\text { (Canada, Bancroff) }\end{array}$ & $0.0000544(51)$ & $0.0072560(32)$ & $1.59136(67) \times 10^{-8}$ \\
\hline $\begin{array}{l}\text { Mistamisk } \\
\text { (Canada, Labrador) }\end{array}$ & $0.0000544(46)$ & $0.0072553(39)$ & $8.145183(59) \times 10^{-9}$ \\
\hline \multicolumn{4}{|c|}{$\begin{array}{l}\text { (Analytical uncertainties refer to the least digit significant figures.) } \\
\text { value. The instrument detection limit for the ratio }{ }^{236} \mathrm{U} /{ }^{238} \mathrm{U} \text { was calculated as three times the standard } \\
\text { uncertainty of the background rate divided by the typical }{ }^{238} \mathrm{U} \text { intensity }{ }^{238} \mathrm{U} \cong 2 \times 10^{-11} \mathrm{~A} \text { ). This yield a } \\
\text { value for the detection limit of } 1.2 \times 10^{-10} \text {. Some }{ }^{236} \mathrm{U} \text { has been found in exceptional natural samples e.g. } \\
\text { Oklo reactor. }\end{array}$} \\
\hline
\end{tabular}

Table 3. Isotope ratios of uranium measured from soil samples

\begin{tabular}{llll}
\hline $\begin{array}{l}\text { Sample } \\
\text { Name }\end{array}$ & ${ }^{234} \mathrm{U} /{ }^{238} \mathrm{U}\left( \pm 2 \sigma_{\mathrm{m}}\right)$ & ${ }^{235} \mathrm{U} /{ }^{238} \mathrm{U}\left( \pm 2 \sigma_{\mathrm{m}}\right)$ & ${ }^{236} \mathrm{U} /{ }^{238} \mathrm{U}\left( \pm 2 \sigma_{\mathrm{m}}\right)$ \\
\hline JLK-1 & $0.0000546(51)$ & $0.0072560(32)$ & Not detectable \\
Chernobyl A & $0.000090(46)$ & $0.008194(39)$ & $0.000325(45)$ \\
Chernobyl B & $0.000117(74)$ & $0.00993(43)$ & $0.000972(44)$ \\
\hline
\end{tabular}

(Analytical uncertainties refer to the least digit significant figures.)

The isotopic measurement of uranium from two surface soil samples from Chernobyl exclusion zone has been given in Table 3 with the objective of detecting the presence and potential source of anthropogenic uranium. The isotopic composition of Chernobyl soil samples differed from rest samples with reference to ${ }^{234} \mathrm{U}$ and ${ }^{236} \mathrm{U}$ isotopes and confirms the presence of non-natural ${ }^{236} \mathrm{U}$ from the radioactive fallout. This shows the heterogeneity in the distribution of isotopically enriched of isotopically depleted material within the soil.

\subsection{CONCLUSIONS}

We have developed a procedure to measure ${ }^{234} \mathrm{U} /{ }^{238} \mathrm{U},{ }^{235} \mathrm{U} /{ }^{238} \mathrm{U}$ and ${ }^{236} \mathrm{U} /{ }^{238} \mathrm{U}$ isotope ratios to a better than $0.2 \%$ at two standard deviation (sd). This method should be applicable to a wide range of environmental and geological problems which are limited by sample size or for which higher precision is required. However, while measuring ${ }^{236} \mathrm{U} /{ }^{238} \mathrm{U}$ ratio, the possibility of distinguishing between different samples is limited by the instrument detection limit. Therefore, the natural abundance of ${ }^{236} \mathrm{U}$ may be sill lower than that detected by the present instrument. 


\section{Acknowledgement}

One of us (SKS) is highly grateful to President of NIRS in funding the upgradation of TIMS to be equipped with WARP energy filter. Sincere thanks to Pofessor H. Hidaka for providing uranium ore samples and for valuable discussions.

References

1. V.G. Baryakhtar, I.M. Vyshnevs'ky, in Chornobyl Catastrophe, edited by V.G. Baryakhtar (Export of Ukraine, Kyiv, 1997).

2. G. Friedlander, J.W. Kennedy, E.S. Macias, J.M. Miller, Nuclear and Radiochemistry, John Wiley and Sons, New York (1981).

3. M. Ivanovich, R.S. Harmon, Uranium Series Disequilibrium: Applications to Environmental Problems, Clarendon Press, Oxford (1993).

4. X. Z. Luo, M. Rehkämper, D.C. Lee, A.N. Halliday, Int. J. Mass Spectrom. Ion Processes, 171, 105 (1997)

5. S.K. Sahoo, A. Masuda, Proc. Japan Acad., 76, 151 (2000).

6. I. Croudace, P. Warwick, R. Taylor, S. Dee, Anal. Chim. Acta, 371, 217 (1998).

7. Z.A. Palacz, P.A. Freedman, A.J. Walder, Chem. Geol., 101, 157 (1992). 